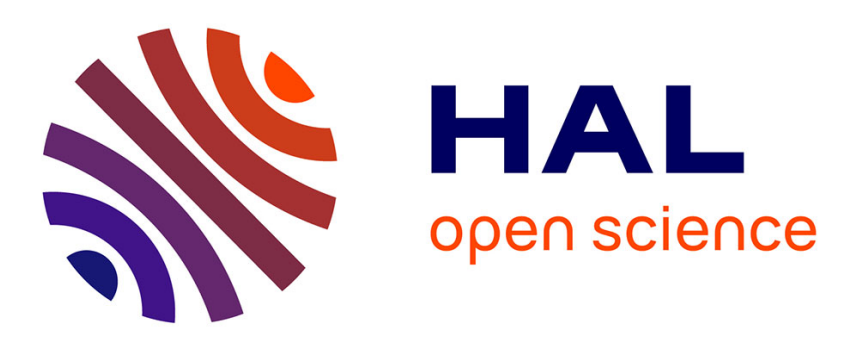

\title{
Anisotropic sintering behavior of freeze-cast ceramics by optical dilatometry and discrete-element simulations
}

Aaron Lichtner, Denis Roussel, Daniel Röhrens, David Jauffres, Julie

Villanova, Christophe L. Martin, Rajendra K Bordia

\section{To cite this version:}

Aaron Lichtner, Denis Roussel, Daniel Röhrens, David Jauffres, Julie Villanova, et al.. Anisotropic sintering behavior of freeze-cast ceramics by optical dilatometry and discrete-element simulations. Acta Materialia, 2018, 155, pp.343-349. 10.1016/j.actamat.2018.06.001 . hal-01864376

\section{HAL Id: hal-01864376 https://hal.science/hal-01864376}

Submitted on 29 Aug 2018

HAL is a multi-disciplinary open access archive for the deposit and dissemination of scientific research documents, whether they are published or not. The documents may come from teaching and research institutions in France or abroad, or from public or private research centers.
L'archive ouverte pluridisciplinaire HAL, est destinée au dépôt et à la diffusion de documents scientifiques de niveau recherche, publiés ou non, émanant des établissements d'enseignement et de recherche français ou étrangers, des laboratoires publics ou privés. 


\title{
Anisotropic Sintering Behavior of Freeze-Cast Ceramics by Optical Dilatometry and Discrete-Element Simulations
}

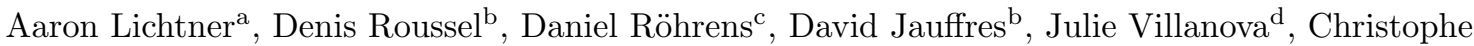 \\ L. Martin ${ }^{\mathrm{b}}$, Rajendra K. Bordia ${ }^{\mathrm{e}}$ \\ ${ }^{a}$ Dept. of Mat. Sci. and Eng., University of Washington, Seattle, Washington, USA \\ ${ }^{b}$ Universite de Grenoble Alpes, CNRS, SIMaP, F-38000 Grenoble, France \\ ${ }^{c}$ Forshunzentrum, Institutfr Energie und Klimaforschung, D-52425 Jülich, Germany \\ ${ }^{d}$ ESRF, The European Synchrotron CS 40220 38043 Grenoble Cedex 9, France \\ ${ }^{e}$ Dept. of Mat. Sci. and Eng., Clemson University, Clemson, South Carolina, USA
}

\begin{abstract}
Directional freeze-casting of ceramic slurries followed by freeze drying and partial sintering results in materials with highly anisotropic properties parallel and transverse to the freezing direction. Physical measurements and optical dilatometry confirm that, during sintering, freeze-cast structures experience more strain along their freezing direction than transverse to it. Discrete Element (DEM) simulations of equivalent freeze-cast structures confirm this behavior. These simulations indicate that not only is sintering anisotropic on the macroscopic scale but within the walls and macropores themselves. It was determined that the anisotropic particle contact network that resulted from the aligned macropores led to anisotropic shrinkage during sintering.
\end{abstract}

Keywords: Freeze-Cast, Anisotropic Pores, Optical Dilatometer, Sintering, Discrete Element Simulations

\section{Introduction}

Freeze-casting is a materials processing technique that can be used to create a variety of anisotropic porous microstructures for use in fields such as materials for energy conversion devices [1], dentistry [2], bioengineering [3], and even food science [4]. This technique is attractive because of the high level of control and wide range of properties one can achieve with relatively simple equipment and primarily "green" methods. A significant number of researchers have reported 
on the effects of processing and microstructure on properties [5, 6] but little has been published concerning the actual sintering process of these highly anisotropic, porous structures. It is nonetheless an important topic of fundamental and practical interest. Freeze casting provides an interesting case study of the impact of a strongly anisotropic initial green microstructure on the sintering behavior. The shrinkage during sintering is an important fundamental outcome of sintering theories and models. It is well known that the shrinkage is a strong function of the microstructure. Thus, it is expected that these highly anisotropic microstructures will have anisotropic shrinkage. This study provides a quantitative confirmation of this and provides particle scale insights on the causes of this anisotropic shrinkage. From a practical point of view, the manufacturing of sintered parts with designed geometry requires in-depth understanding and modeling of the shrinkage during sintering and its dependence on the green microstructure.

Farhangdoust et al. [7] reported strains in both directions after sintering hydroxyapatite and found that their samples shrunk less in the freezing direction than in their transverse direction. They argue that there is more empty space, spatially speaking, in the transverse direction so sintering will occur to a higher extent. However, it is generally admitted and observed that shrinkage is more pronounced in the direction of pore elongation (i.e. the radial direction for a uniaxially pressed sample) 8]. Based on this commonly held belief, a freeze-cast sample would shrink more in the freezing direction, in apparent contradiction with the observation reported by Farhangdoust et al..

In this paper, we report on the in-situ sintering of freeze-cast composite porous ceramics with aligned macropores for use as solid oxide fuel cell (SOFC) cathodes. The fabrication process, described in 1], consists of directionally freezing a well-dispersed, aqueous ceramic slurry. Due to the directional temperature gradient and anisotropic crystallographic nature of ice, long continuous ice channels form within the suspension, consolidating the ceramic particles into the interlamellar spaces between ice crystals. Subsequent sublimation of the ice leads to a green body with macropores where the ice crystals had been and micropores between the ceramic particles of the green body. Sintering is then typically performed to consolidate the green body enough to provide adequate strength while maintaining a high degree of reactive surface area [9, 10].

To investigate the effects of the anisotropic microstructure on sintering behavior, an opti- 
cal dilatometer (TOMMIplus, Fraunhofer ISC, Wertheim, Germany) was used. This is a hightemperature furnace equipped with two aligned quartz windows on either side of the sintering sample (Fig. 1). At one quartz window is a high-powered light source and at the other is a highspeed camera with a telecentric lens. The light source creates a silhouette of the sample that is captured by the camera. Software is employed to track the edges of the silhouetted sample as it expands or contracts over time [11. Since an entire face of the sintering sample can be observed, the strains in multiple directions can be tracked simultaneously making this technique ideal for anisotropic or irregularly shaped samples compared to conventional contact dilatometry.

3D Discrete Element Method (DEM) simulations were also performed to confirm the sintering behavior observed experimentally and support the discussion. Real 3D freeze-cast structures, obtained from high resolution synchrotron-based X-Ray tomography, were used [12].

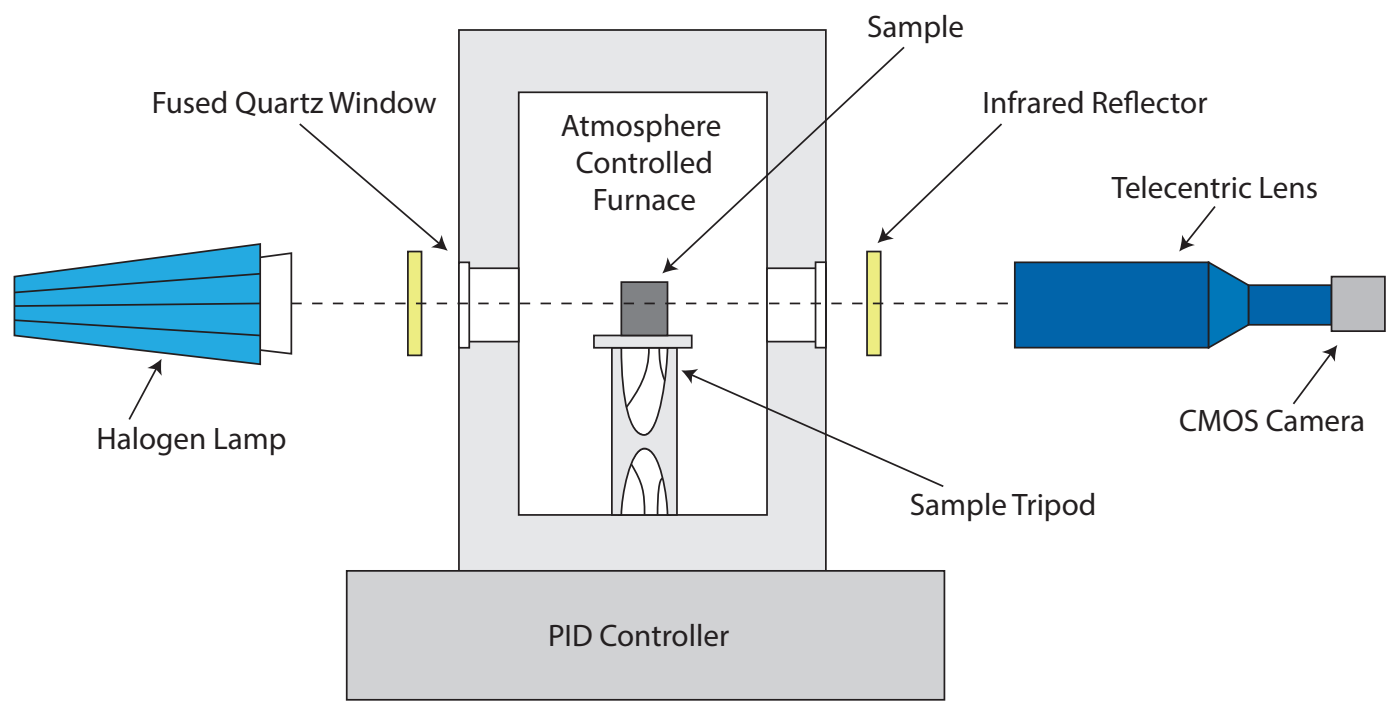

Figure 1: Schematic of the inner-workings of an optical dilatometer. Freeze-cast samples were placed so that the freezing direction (longitudinal) was pointing up. 


\section{Methods}

\subsection{Optical Dilatometry}

Freeze-cast specimens with 80 vol. \% green-state total porosity were made as per [9, 10] but only sintered to $1000{ }^{\circ} \mathrm{C}$. This step results in a small amount of neck growth, providing strength for subsequent handling, but a negligible amount of densification, as was confirmed by physical measurement $(<0.01$ strain). All specimens were made from a 40:60 volume ratio mixture of Lanthanum Strontium Manganite to Yttria-Stabilized Zirconia (LSM-YSZ), a mixture appropriate for use as an SOFC cathode. The porous ceramics were infiltrated with a low-viscosity resin (EpoHeat, Buehler GmbH, Irvine, CA) and sectioned into cuboidal specimens approximately $8 \mathrm{x}$ $8 \times 12 \mathrm{~mm}$ with the longest edge of the freeze-cast samples aligned with the freezing-direction (longitudinal). This operation, similar to what was done in [10], ensured that only the steady-state anisotropic regions of the freeze-casts were used for dilatometry experiments. After sectioning, the specimens were measured precisely then heated to $500{ }^{\circ} \mathrm{C}$ at $2{ }^{\circ} \mathrm{C} \mathrm{min}-1$ and held there for 2 hours until the resin had completely burned out.

The fragile samples, now devoid of any organic residue, were placed in the optical dilatometer with their freezing direction pointing up. During testing, the samples were sintered to $1400{ }^{\circ} \mathrm{C}$ at a rate of $10^{\circ} \mathrm{C} \min ^{-1}$ in air. The optical dilatometer camera captured an image each minute by averaging a total of 10 images to reduce noise.

\subsubsection{Data Analysis}

For each sample tested, the series of images produced by the optical dilatometer were thresholded into binary images. Then, using a plugin written for ImageJ, black pixels in both the vertical and horizontal directions were counted at predetermined locations giving both the longitudinal and transverse strains as a function of time and temperature. On average, both longitudinal and transverse strains were measured at three distinct locations for each sample. The curves were then fitted with a gaussian smoothing algorithm to reduce noise.

\subsection{Discrete-Element Simulations}

DEM simulations are based on the sintering model used in [13, 14]. This model originates from the early models proposed by Parhami and McMeeking [15], which are well adapted for systems 


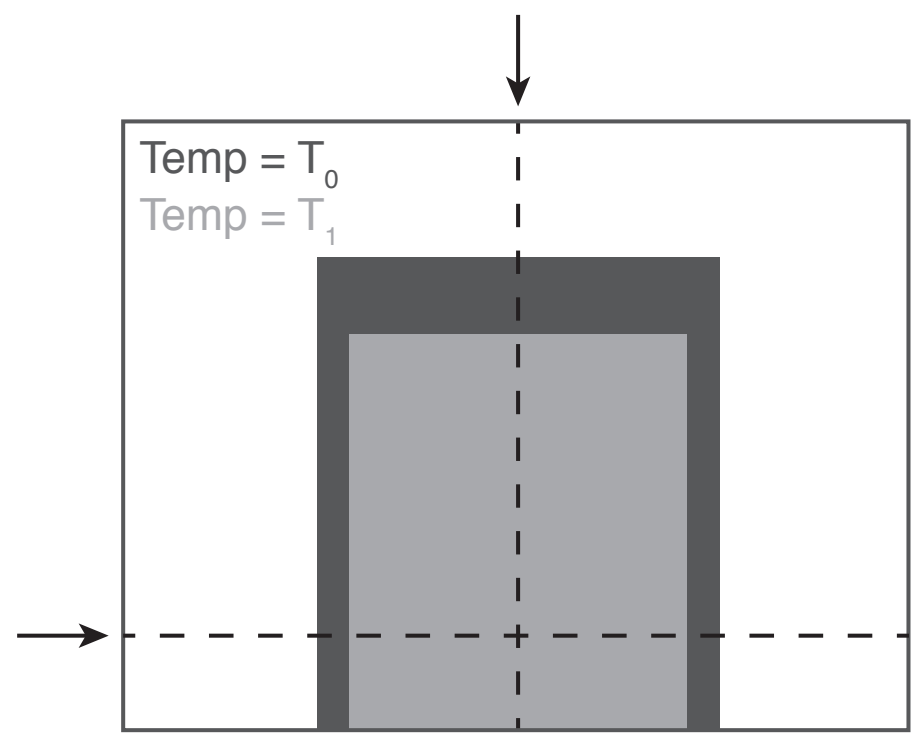

Figure 2: Schematic illustrating how strains are determined from images in multiple directions. The figure shoes the sample at the initial temperature, $T_{0}$ (black) and the sample at the sintering temperature, $T_{1}$ (grey).

where grain boundary and surface diffusion are the major mechanisms of mass transport. In DEM, particles rearrange due to the action of sintering forces at contacts with neighbouring particles. For this study it should be made clear that we are interested in the evolution of the freeze-cast microstructures with and without macropores, not in the kinetics of sintering.

Particles are modeled as spheres interacting dynamically with each other through their contacts. The diffusion parameter $\Delta_{b}$ is modeled as:

$$
\Delta_{b}=\frac{\Omega}{k T} \delta_{b} D_{b}
$$

where $\Omega$ is the atomic volume and $D_{b}=D_{0 b} \exp \left(-Q_{b} / R T\right)$ is the diffusion coefficient for vacancy transport in a grain boundary with thickness $\delta_{b}$ and activation energy $Q_{b}$. For two spherical particles of identical radius $R$, having a contact radius $a_{s}$ and particle overlap $h$, the normal force, 
$N_{s}$ between the particles is given by:

$$
N_{s}=\frac{\pi a_{s}^{4}}{8 \Delta_{b}} \frac{\mathrm{d} h}{\mathrm{~d} t}-\frac{9}{8} \pi R \gamma_{s}
$$

where the surface energy between the particles is denoted as $\gamma_{s}$. Tangential contact forces are neglected in these simulations since we assume that contacts are large enough to oppose any rotation of particles.

The contact radius simply grows according to Coble's geometric model [16]:

$$
\frac{\mathrm{d} a_{s}}{\mathrm{~d} t}=\frac{R}{a_{s}} \frac{\mathrm{d} h}{\mathrm{~d} t}
$$

For a multimodal packing as modeled here, we define $R^{*}$ as the equivalent radius for two particles of radii $R_{1}$ and $R_{2}$ :

$$
R^{*}=R_{1} R_{2} /\left(R_{1}+R_{2}\right)
$$

As suggested by Parhami et al. [17, the model of Bouvard and McMeeking may be generalized for contacts between particles of different sizes by replacing $R$ in equations $(2)$ and $(3)$ by $2 R^{*}$.

For this study, two numerical structures were generated, one with realistic freeze-cast macropores and one without. To do so, two identical packings of particles were generated with 50 vol. $\%$ total porosity, typical for green bodies without macroporosity. A bimodal size distribution corresponding to the 40:60 vol\% LSM:YSZ ratio was used with particle sizes of $0.5 \mu \mathrm{m}$ (YSZ) and $1 \mu \mathrm{m}$ (LSM). A 3D freeze-cast structure was reconstructed from a synchrotron X-ray characterization of one of our samples [12]. The voxel resolution for this image was approximately $75 \mathrm{~nm}$, while the field of view was $\sim 90^{3} \mu \mathrm{m}^{3}$. The image was merged with one of these DEM particle packings to produce macropores in addition to the already present interparticle pores. The DEM structure from the synchrotron reconstructed image contained approximately 208,000 YSZ particles and 17,000 LSM particles. The average initial coordination number was 3.1 in the overall structure and 3.8 inside the particle-packed regions. The procedure to generate the DEM structure from the synchrotron reconstructed image has been described in more detail in [18. Both DEM structures, with and without macropores, were then sintered numerically until a density of 0.75 was reached within the particle-packed regions, hereafter called the walls. The intrawall density of the simulated structure 
was equivalent to that of a physical LSM-YSZ freeze-cast sintered to $1400{ }^{\circ} \mathrm{C}$.

It is important to note that since the size of the simulated structures is so much smaller than the physical ones (typical experimental samples are tens of $\mathrm{mm}$ in size, numerical sample are only $90 \mu \mathrm{m}$ per side), there are some important differences in their overall structures. Large scale freeze-casts are composed of colonies of lamellar pores which are randomly oriented in the $\mathrm{x}-\mathrm{y}$ plane [10]. The simulated freeze-casts contain only those pores within a single colony, consequently, they lose the isotropic nature of the transverse direction seen in the experimental samples. The result of this is that physical samples have two distinct microstructural and behavioral directions (longitudinal and transverse) while the DEM samples have three (longitudinal, transverse-align, and transverse-non-align). The behavior of the transverse direction in physical freeze-casts will be some linear combination of the transverse-align and transverse-non-align direction observed in the DEM samples.

\section{Results}

\subsection{Experimental Sintering Behavior}

Sintering studies of 40:60 vol. \% LSM-YSZ show that densification starts to occur at approximately $1000{ }^{\circ} \mathrm{C}$ and continues until full densification is achieved at approximately $1500{ }^{\circ} \mathrm{C}$. The vast majority of densification occurs in the $1100-1300{ }^{\circ} \mathrm{C}$ range. The optical dilatometer used in this study was limited to a $1400{ }^{\circ} \mathrm{C}$ maximum, which despite not capturing the full sintering profile, does capture the majority of densification. Figure 3 shows the aggregated sintering profiles from multiple freeze-casts. One can see that the longitudinal and transverse strains begin to deviate at approximately $1200{ }^{\circ} \mathrm{C}$, with the longitudinal direction shrinking to a greater extent, on average, than the transverse one.

Physical measurements of the porous freeze-casts before and after sintering to $1400{ }^{\circ} \mathrm{C}$ clearly show the anisotropic sintering behavior experienced by the freeze-casts in the two testing directions

(Fig. 4). In the longitudinal direction, on average there was an additional shrinkage of $3.4 \%$ compared to the tranverse direction. This is contradictory to the results of Farhangdoust et al. [7] who claim that sintering strains are enhanced in the transverse direction, not the longitudinal one. 


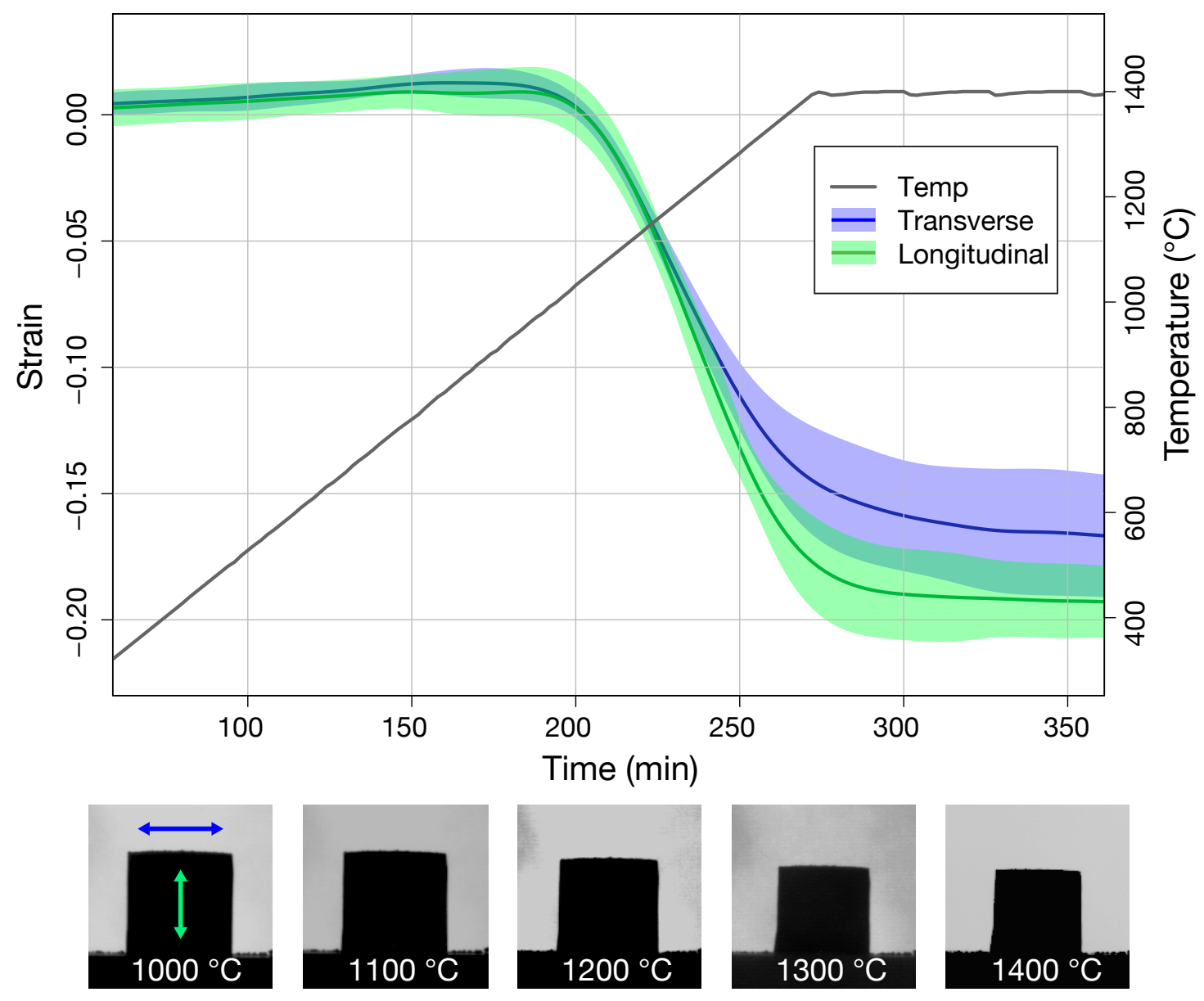

Figure 3: Aggregated sintering profiles from samples oriented with their freezing-direction up (longitudinal). Stillframes from a freeze-cast sintering run are included for reference. A minimum of 15 measurements were done for each curve.

In our experiments, cuboid samples were cut so as to exclude the initial, isotropic cellular structure found at the bottom that may have influenced the sintering behavior seen by Farhangdoust et al.. In addition, by doing so, the continuous skin that generally forms on the side of a freeze-casted cylinder is also removed. The presence of such a continuous skin may have promoted the transverse shrinkage seen by Farhangdoust [7]. 


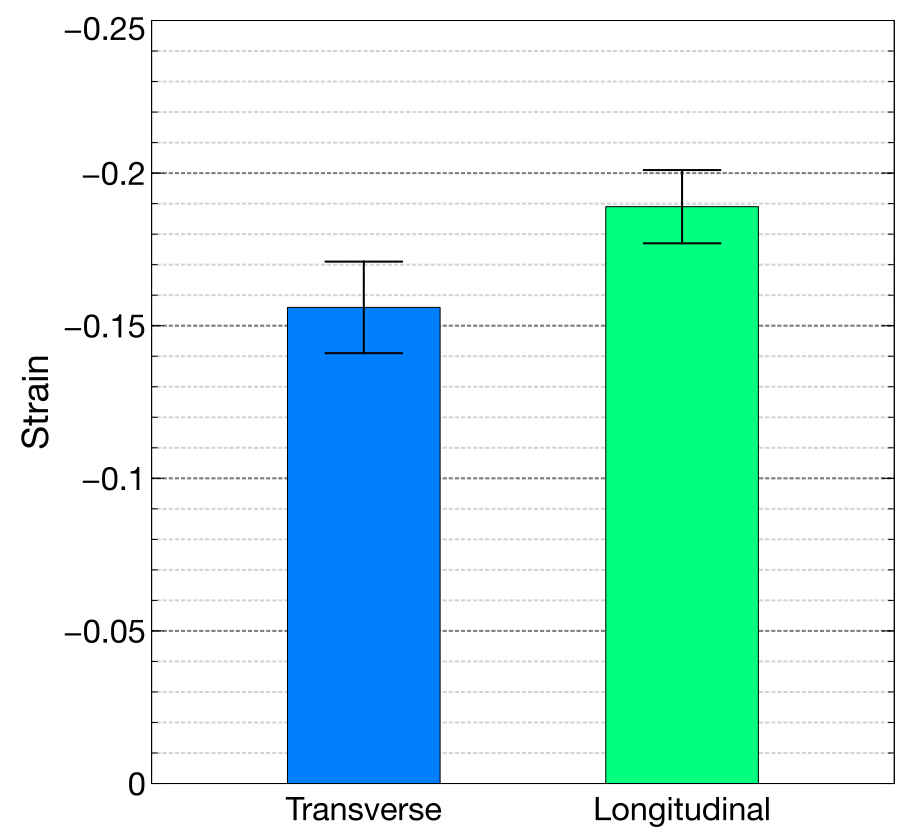

Figure 4: Sintering shrinkage from averaged physical measurements taken before and after sintering freeze-casts for sintering temperatures of $1400{ }^{\circ} \mathrm{C}$ in air. A minimum of 5 samples were utilized per treatment.

\subsection{Simulated Sintering Behavior}

DEM simulations were performed on particle packings with and without macroporous structures. For simulations performed on structures with realistic freeze-cast macropores, plotting the strains in the $\mathrm{x}, \mathrm{y}$ and $\mathrm{z}$-directions one can see that there is one direction that shrinks to a lesser extent $(\sim 3$ \%) than the other two (Fig. 5). As expected, this direction, y, is normal to the freezing-direction. The microstructure along this direction (Transverse-non-align) is most similar to the transverse direction in physical samples which also saw lower strain values during sintering (Fig. 5).

Note, that the x-direction (transverse-align) sees equivalent strain values to the freezing direction (z), illustrating that within a colony of pores, the properties in the freezing-direction are similar to those in the transverse-align direction. The quantitative comparison between the macroscopically measured strain (Transverse: $-0.155 \pm 0.015$, Longitudinal: $-0.19 \pm 0.01$ ) and the DEM simulations $(y:-0.1, x, z:-0.14)$ shows that DEM simulations underestimate strains. This may be due to the size of the DEM representative volume obtained from the X-ray tomography which may be 

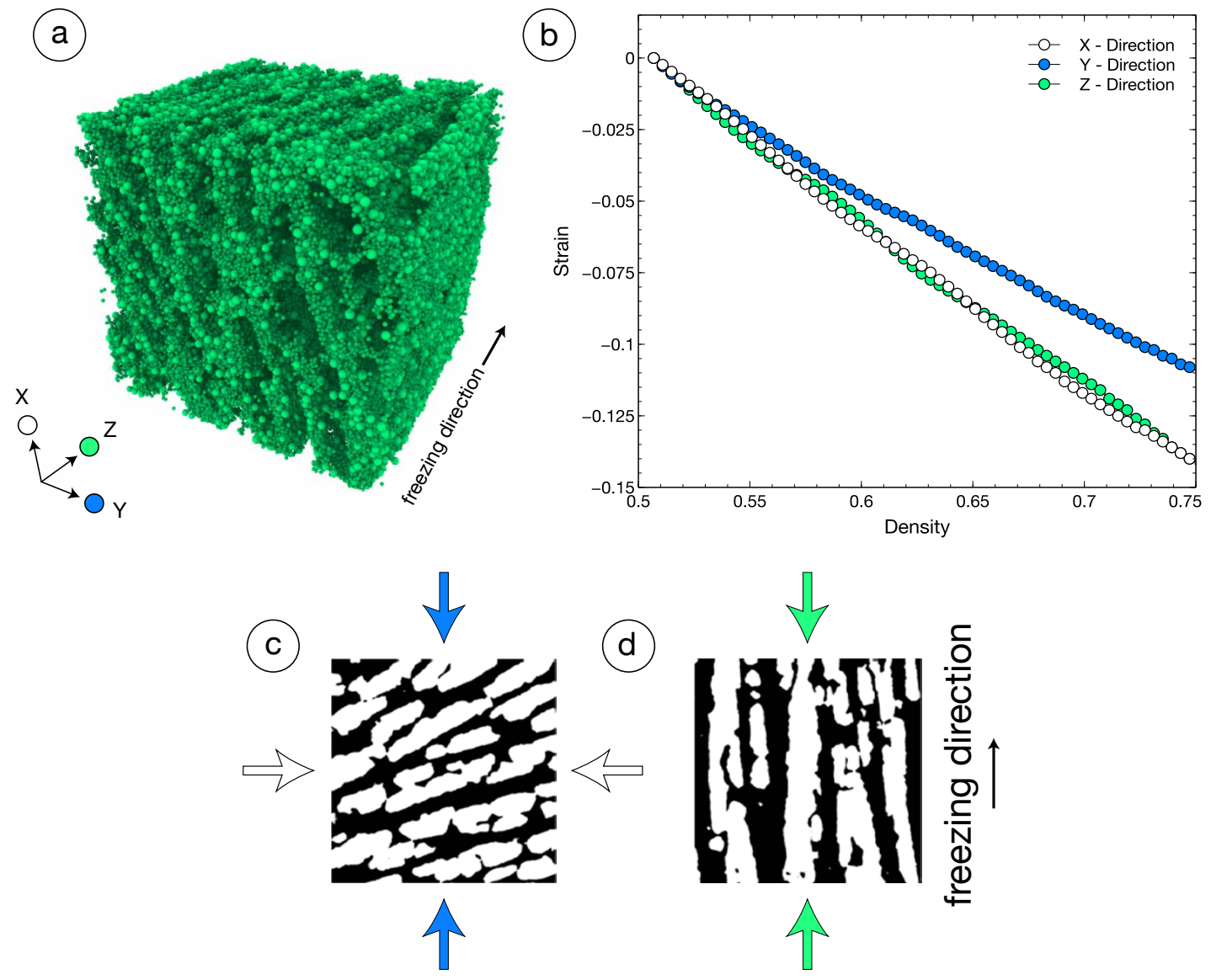

Figure 5: (a) Simulated sintering of a freeze-cast DEM structure $\left(\sim 90^{3} \mu \mathrm{m}^{3}\right)$ showing (b) anisotropic shrinkage. Also shown are the (c) x-y and (d) z-y cross-sections to illustrate the macrostucture and relevant shrinkage. Macropores are shown in white.

too small to fully model the behavior seen in physical samples. Still, we note that the $\sim 90^{3} \mu \mathrm{m}^{3}$ freeze-cast DEM structure captures approximately both the strain magnitude and the ratio between the transverse and the longitudinal strains.

As a comparison, the sintering strain experienced by the DEM packing without macropores is plotted alongside strain values from the sintered freeze-cast DEM structure (Fig. 6). We show here that the freeze-cast structure has an unequivocal effect on the sintering process. 


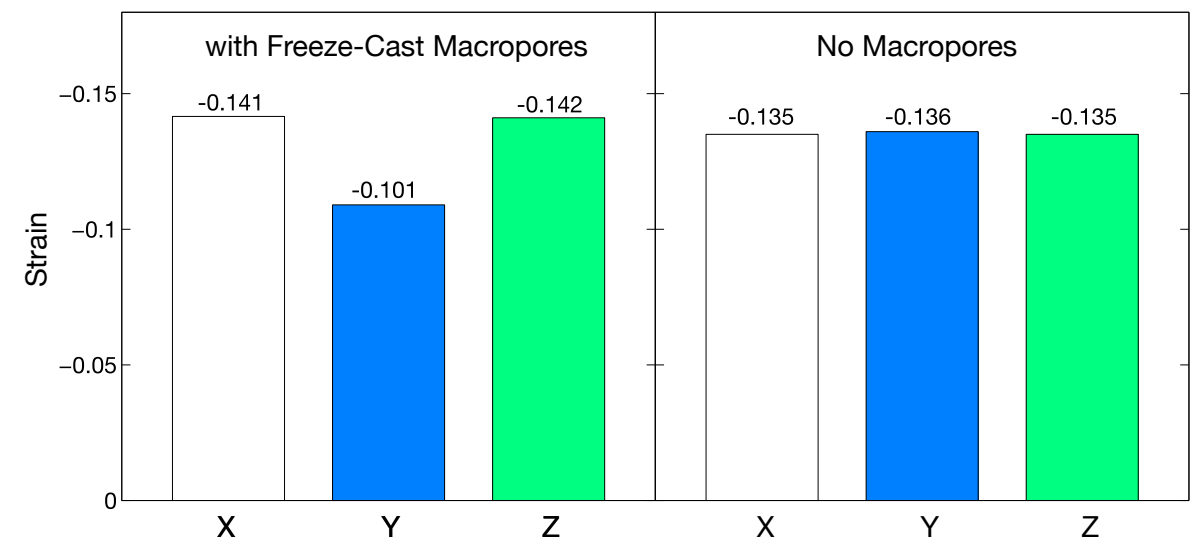

Figure 6: Strain values in each direction at the final density of 0.75 for DEM simulated structures with and without macropores.

\section{Discussion}

Freeze-cast LSM-YSZ underwent anisotropic densification during sintering. Experimentally, samples shrunk to a greater extent $(3-4 \%)$ longitudinally (freezing direction) compared with their transverse directions when sintered to $1400{ }^{\circ} \mathrm{C}$. Discrete element sintering simulations of equivalent particle packings confirm that the presence of macropores contained within the freezecast is responsible for the anisotropic behavior (Fig. 6).

To complement the anisotropic, macroscopic DEM strains seen in Fig. 5 we took strain measurements in between pairs of specified particles of the DEM numerical structure. These investigations provide additional understanding of how sintering evolves at the local level within freeze-cast structures. Fig. 7 shows the evolution of these local strains as a function of relative density. We chose to measure the strains in the walls in the $x, y, z$ directions and across two walls (i.e. the strain in a macropore) in the $y$ direction. Several points were chosen to evaluate the dispersion accompanying these strain measurements. Fig. 7 shows that the local strains in the $y$ direction, be it in the walls, $\epsilon_{\text {wall }(y)}$, or in the macropores, $\epsilon_{\text {macropore(y) }}$, are on average, smaller than the strains in the wall in the $x$ or in the $z$ directions $\left(\epsilon_{\text {wall }(x)}, \epsilon_{\text {wall }(z)}\right)$. This is consistent with the macroscopic strains that have been measured at the scale of the whole freeze-cast DEM structure (Fig. 5). Strains in Fig. 
7 are of course distributed since they characterize strains in between pairs of discrete points in the structure. Interestingly, the strains in the $y$ direction are similar in the solid wall and in the macropore, demonstrating that the freeze-cast structure shrinks homogeneously in the transverse-non-align direction, albeit to a lesser extent than in the transverse-align direction.
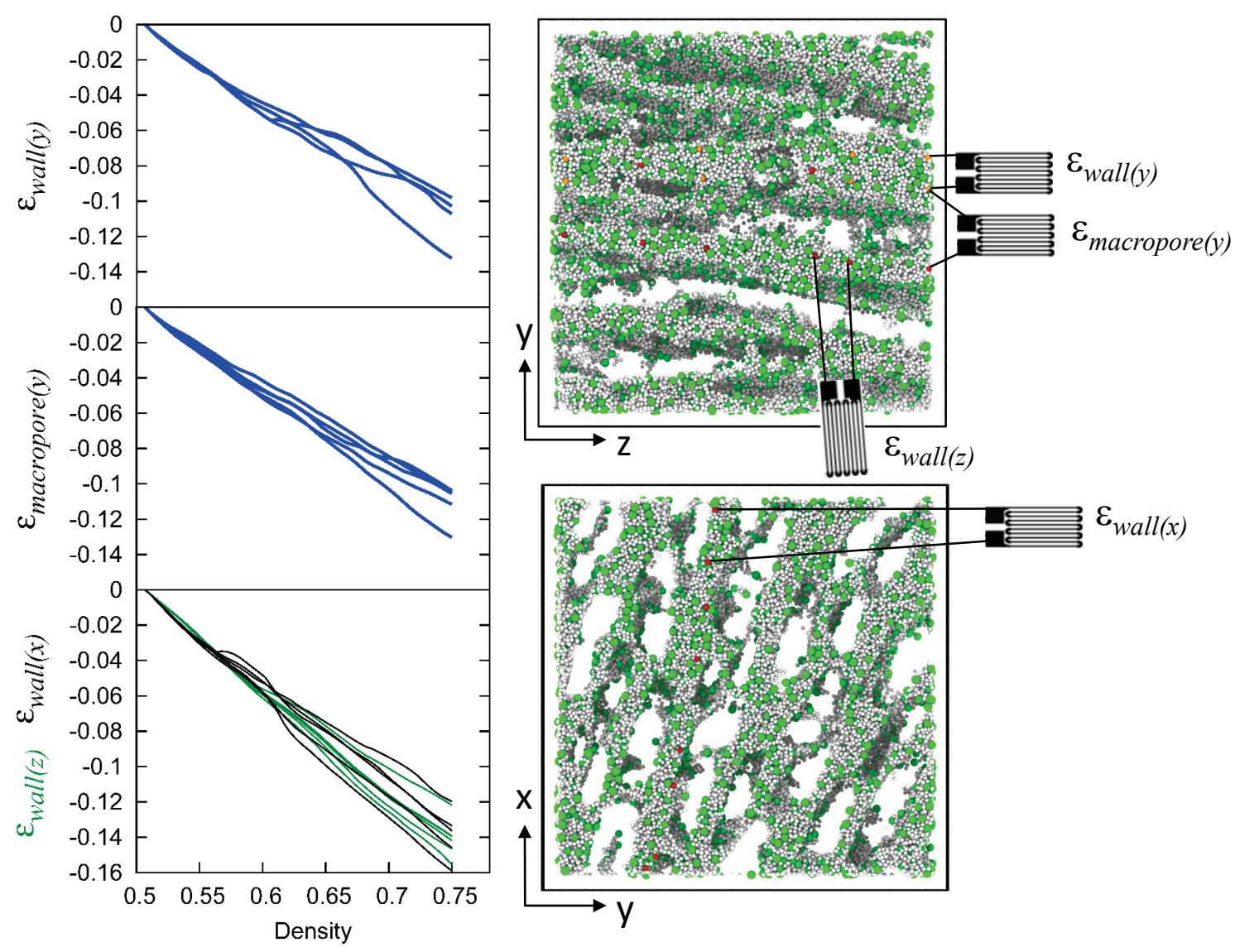

Figure 7: Evolution of strains in the walls $\left(\epsilon_{\text {wall }}\right)$ and in the macropores ( $\left.\epsilon_{\text {macropore }}\right)$ at selected pairs of particles used as strain gauges.

As another level of investigation, we can examine the coordination number of particles in a DEM structure after sintering. Areas which are more densely packed will have densified more substantially than others. Looking at results of the discrete element sintering simulations in figure 8 , one can see that the regions of highly coordinated particles run along the $\mathrm{x}$ and $\mathrm{z}$-directions. These are both 
the directions where greater overall shrinkage values were observed. Comparatively, the y-direction is highly discontinuous, with only small particulate bridges connecting walls. In addition these bridges have experienced lower densification than the walls as revealed by their lower coordination numbers.

Tanaka et al. [19] demonstrated that the anisotropy of the contact orientation network and its influence on sintering may be quantitatively rendered by studying $2 \mathrm{D}$ images of die-pressed compacts. Alternatively, using the full 3D information from our DEM structure, the stereographic projections of the contact orientation network can be obtained. Fig. 9 a shows that indeed in the center of the projection, there is a lack of contacts whose normals are preferentially oriented in the $y$ direction (as indicated by the yellow color in the center of the pole figure). This lack of contacts with normals oriented in the $y$ direction is not immediately obvious upon observing the stereographic projection. Still, it is confirmed by Fig. 9b, which shows the histogram of contact angles with respect to the $y$ axis. As the contact angle increases from $\theta=0$, a gradual increase in the number of occurrences is clearly indicated.

The anisotropy shown in Fig. 9 is not pronounced because it is only the particles that are found along free surface of the walls that lead to a macroscopic contact anisotropy (the contacts within the walls themselves are still isotropically distributed). Still this anisotropy in contact orientation is sufficient to lead to the observed anisotropic shrinkage. It also confirms the qualitative information given by the coordination number map in Fig. 8 ,

Sintering is a mass-transport phenomena, it is driven by the system's desire to reduce surface area and controlled by mass diffusion. Particles that are well within the walls of a freeze-cast are surrounded in all directions by particles and so will sinter isotropically to reduce that energy. Particles which find themselves running along the free surfaces of macropores are only partially surrounded by other particles (Fig. 8). Because of this they will experience a lower sintering driving force. In other words, the lack of contacts whose normals are oriented along the $y$ direction (Fig. 9), will result in enhanced shrinkage along the the walls ( $x$ and $z$ ). 


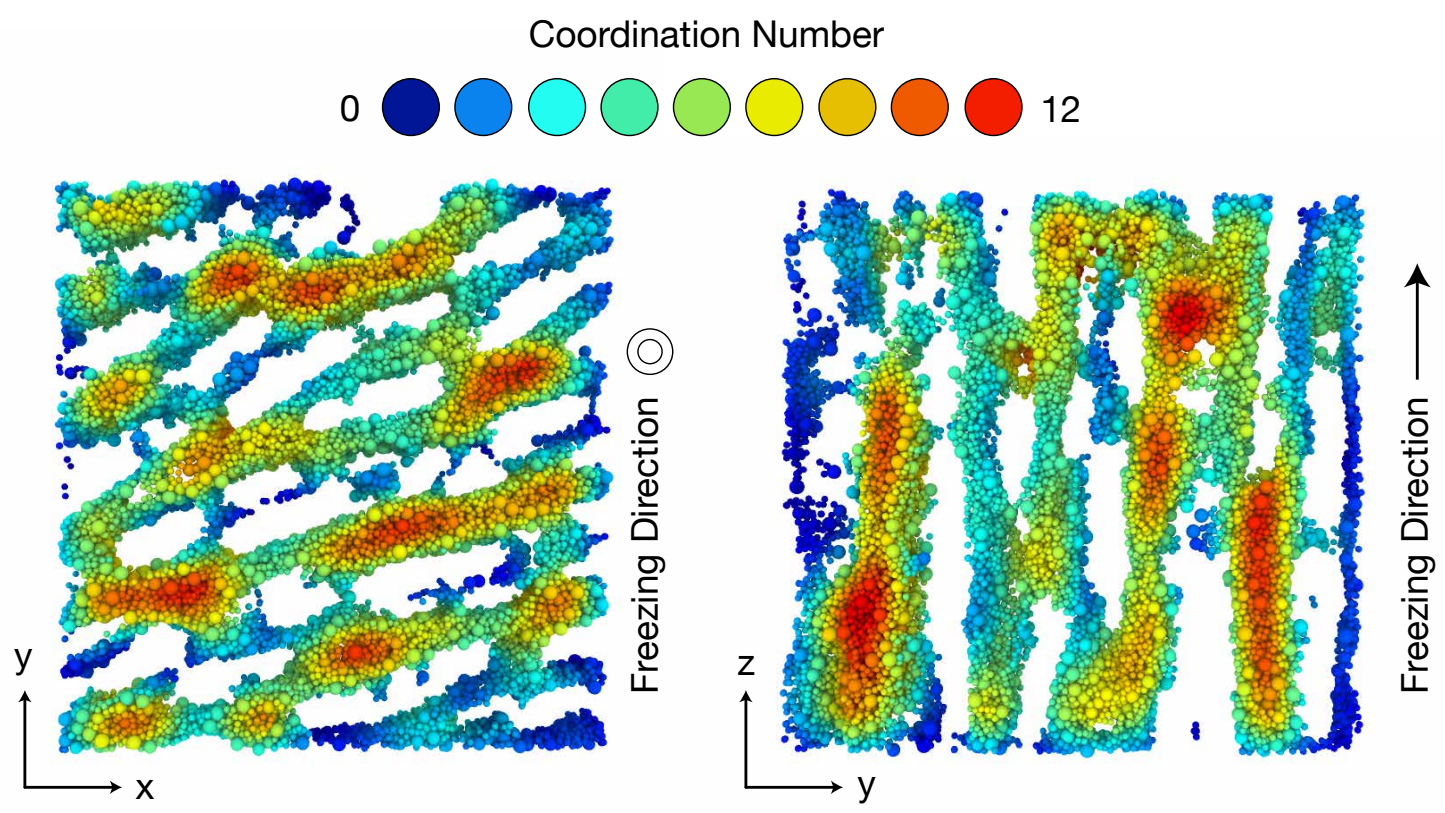

Figure 8: Coordination analysis of particles in a sintered DEM structure.

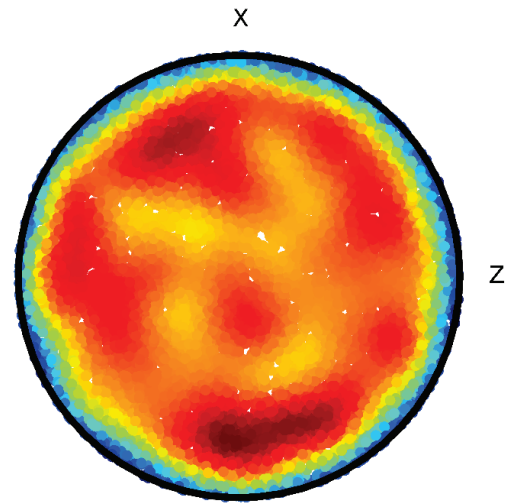

a

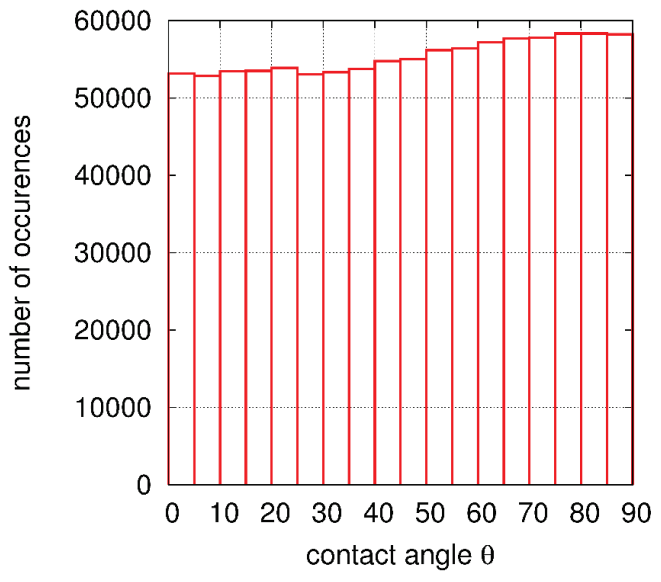

b

Figure 9: Anisotropy of contact orientation. a) Stereographic projections of the contact orientation network in the $(x, z)$ plane from the unsintered DEM structure. Colors indicate relative density in arbitrary units. b) Histogram of contact angles with respect to the $y$ axis. The number of occurrences is normalized by $2 \pi(\cos (\theta)-\cos (\theta+\delta \theta))$ for each bin. 


\section{Conclusions}

Sintering of freeze-cast structures resulted in significantly different relative levels of shrinkage in the longitudinal and transverse directions. As shown by optical dilatometry measurements and DEM simulations, the longitudinal direction shrinks more than the tranverse one ( $~ 3-4 \%$ more). Localized strain measurements between pairs of particles within DEM simulations indicate that not only do freeze-cast samples shrink anisotropically on the macro-scale but do so at the scale of the walls and macropores themselves. Stereographic projection of the particle contact orientation network from the unsintered sample suggests that the freeze-casting process reduces the number of contacts with specific orientations. The relatively lower ratio of particle contacts with normals aligned along the transverse direction results in less shrinkage normal to the freezing direction Reduced densification of the particulate bridges that connect walls, as compared to the walls themselves, is confirmed by DEM results. The orientation of free surfaces and the fact that, spatially there is more void space in the transverse direction (macropores) favors anisotropic shrinkage. This study demonstrates that near netshape sintering of porous anisotropic parts might not be straightforward even for simple geometries. Thus, the successful forming of porous anisotropic ceramics requires a detailed investigation and further modeling of sintering behavior.

\section{Acknowledgments}

This work was supported by the U.S. National Science Foundation (NSF) under grant no. 1008600 and l'Agence de la Recherche under grant no. 2010 BLAM 0931 01. RKB also acknowledges partial support from the NSF under grant no. 1502392. Fig. 9a was built with the help of Alexis Burr using the AITA python toolbox (https://github.com/ThomasChauve/aita).

\section{References}

[1] A. Z. Lichtner, D. Jauffrès, C. L. Martin, R. K. Bordia, Processing of Hierarchical and Anisotropic Porosity LSM-YSZ Composites, Journal of the American Ceramic Society 96 (2013) 2745-2753. 
[2] C. Miller, D. Lath, T. Wang, R. Noort, Freeze Casting of High Strength Composites for Dental Applications, in: Pan European Federation of the International Association for Dental Research, 2008.

[3] S. Deville, E. Saiz, A. P. Tomsia, Freeze casting of hydroxyapatite scaffolds for bone tissue engineering., Biomaterials 27 (32) (2006) 5480-9.

[4] P. T. Nguyen, J. Ulrich, Fast Dispersible Cocoa Tablets: A Case Study of Freeze-Casting Applied to Foods, Chemical Engineering and Technology 37 (8) (2014) 1376-1382.

[5] S. Deville, S. Meille, J. Seuba, A meta-analysis of the mechanical properties of ice-templated ceramics and metals, Science and Technology of Advanced Materials 16 (MAY) (2015) 0.

[6] S. Deville, Freezing Colloids: Observations, Principles, Control, and Use: Applications in Materials Science, Life Science, Earth Science, Food Science, and Engineering, Springer, 2017.

[7] S. Farhangdoust, A. Zamanian, M. Yasaei, M. Khorami, The effect of processing parameters and solid concentration on the mechanical and microstructural properties of freeze-casted macroporous hydroxyapatite scaffolds, Materials Science and Engineering: C 33 (1) (2013) $453-460$.

[8] E. A. Olevsky, Theory of sintering: from discrete to continuum, Materials Science and Engineering: R: Reports 23 (2) (1998) 41-100.

[9] A. Lichtner, D. Jauffrès, D. Roussel, F. Charlot, C. Martin, R. Bordia, Dispersion, connectivity and tortuosity of hierarchical porosity composite SOFC cathodes prepared by freeze-casting, Journal of the European Ceramic Society 35 (2) (2015) 585-595.

[10] A. Lichtner, D. Roussel, D. Jauffres, C. L. Martin, R. K. Bordia, Effect of Macropore Anisotropy on the Mechanical Response of Hierarchically Porous Ceramics, Journal of the American Ceramic Society (2015) 1-9.

[11] F. G. Raether, Current state of in situ measuring methods for the control of firing processes, Journal of the American Ceramic Society 92 (SUPPL. 1) (2009) 146-152. 
[12] J. Villanova, P. Cloetens, H. Suhonen, J. Laurencin, F. Usseglio-Viretta, E. Lay, G. Delette, P. Bleuet, D. Jauffrès, D. Roussel, A. Lichtner, C. L. Martin, Multi-Scale 3D imaging of absorbing porous materials for solid oxide fuel cells, Journal of Materials Science 49 (16) (2014) 5626-5634.

[13] C. L. Martin, L. C. R. Schneider, L. Olmos, D. Bouvard, Discrete element modeling of metallic powder sintering, Scripta Mater. 55 (2006) 425-428.

[14] C. L. Martin, H. Camacho-Montes, L. Olmos, D. Bouvard, R. K. Bordia, Evolution of Defects During Sintering: Discrete Element Simulations, J. Am. Ceram. Soc. 92 (2009) 1435-1441.

[15] F. Parhami, R. McMeeking, A network model for initial stage sintering, Mechanics of Materials 27 (2) (1998) 111-124.

[16] R. Coble, Initial sintering of alumina and hematite, Journal of the American Ceramic Society $41(1958) 55$.

[17] F. Parhami, R. Mcmeeking, A. Cocks, Z. Suo, A model for the sintering and coarsening of rows of spherical particles, Mechanics of Materials 31 (1) (1999) 4361.

[18] D. Roussel, A. Lichtner, D. Jauffrès, J. Villanova, R. K. Bordia, C. L. Martin, Strength of hierarchically porous ceramics : discrete simulations on X-ray nanotomography images, Scripta Materialia 113 (2016) 250-253.

[19] S. Tanaka, Y. Kuwano, K. Uematsu, Packing structure of particles in a green compact and its influence on sintering deformation, Journal of the American Ceramic Society 90 (11) (2007) $3717-3719$. 